\title{
IMPLICACIONES DE LA CALIDAD DE VIDA EN LAS DECISIONES QUIRÚRGICAS DEL CÁNCER DE PULMÓN
}

\author{
Luis Puente-Maestu y Felipe Villar \\ Servicio de Neumología. Hospital General Universitario Gregorio Marañón
}

\section{Resumen}

La evidencia disponible sugiere que los pacientes con un volumen espiratorio forzado en el primer segundo $\left(\mathrm{FEV}_{1}\right)$ mayor de $60 \%$ o en los que se prevé un $\mathrm{FEV}_{1}$ postoratorio mayor de $45 \%$, no desarrollan discapacidad severa tras la cirugía de resección pulmonar; sin embargo, en la actualidad algunos grupos están operando pacientes con EPOC y $\mathrm{FEV}_{1}$ menor de $35 \%$. En pacientes con enfisema puede ocurrir que la función mejore, como en la cirugía de reducción de volumen, pero en los que no es así, hasta un $10 \%$ pueden quedar con insuficiencia respiratoria crónica. Hasta el momento, ninguna prueba funcional o de esfuerzo ha demostrado de forma clara poder predecir quiénes serán estos sujetos o quienes tendrán restricciones importantes en las actividades físicas. Se ha observado que la opinión del equipo médico tampoco es mejor para predecir una calidad de vida postoperatoria pobre. Los supervivientes a largo plazo (más de un año), sin embargo refieren una calidad de vida razonablemente elevada, incluso en los que tienen la función pulmonar reducida. En comparación con otras enfermedades respiratorias crónicas, los supervivientes de cáncer de pulmón parecen estar en mejor situación mental, y en los que tienen una Calidad de vida más baja el origen parece ser principalmente la depresión, y no la limitación física. Por tanto, la evidencia disponible no permite descartar a los pacientes sometidos a cirugía en base a que el equipo suponga una pobre calidad de vida postoperatoria. Además, los estudios que han valorado la QOL sugieren que el paciente que se opera de cáncer de pulmón está dispuesto a asumir el

\section{Abstract}

Available evidence suggests that the patients with a forced expiratory volume in 1 second $\left[F E V_{1}\right]$ over $60 \%$ or in which a postoperative $\mathrm{FEV}_{1}$ is predicted over $45 \%$, they do not develop severe disability after lung resection surgery; nevertheless, currently some groups are operating patients with EPOC and FEV $_{1}$ less than $35 \%$. In emphysema patients can occur that the function improve, as in the volume reduction lung surgery, but in the cases that it does not occur, they can remain with chronic shortness of breath until a $10 \%$. At present, there are not any functional or effort test that be able to predict what patients will present satisfactory residual pulmonary function or chronic shortness of breath or important exercise limitations. It has been noticed that medical team opinion is not satisfactory to predict deficit in postoperative quality of life. Nevertheless, long-term survivors (more than one year) inform relatively good quality of life, even in which they have pulmonary function reduced. In comparison with other chronic respiratory diseases, the survivors of lung cancer seem to be in better psychological state, the deficit in quality of life is caused mainly by depression, and not for physical limitation. Therefore, empirical evidence does not justify excluding undergoing surgery on basis of medical team suppositions about a postoperative poor Quality of life. In addition, studies about Quality of Life suggests patients who is undergo lung cancer surgery are willing to assume risks for their survival, although, they want to receive information about how surgery may affect them.

\section{Correspondencia:}

Servicio de Neumología

Hospital General Universitario Gregorio Marañón

c/ Doctor Esquerdo 4, 28007 Madrid

Email: Ipuente@separ.es 
riesgo con tal de vivir más tiempo, aunque si le gustaría saber cómo le va a afectar la cirugía.

Palabras clave: Cirugía cáncer de pulmón, Calidad de Vida, decisión quirúrgica.
Key words: Cancer lung surgery, Quality of Life, surgery decision.

\section{INTRODUCCIÓN}

Al tomar la decisión de operar a pacientes con cáncer de pulmón, particularmente en mayores de edad o con una función cardiaca o pulmonar reducida, puede surgir el interrogante de si es prudente practicar intervenciones con una mortalidad mayor de lo habitual y con unas probabilidades de curación variable. En estas circunstancias pueden abrirse discrepancias entre médicos, cirujanos, anestesistas, enfermeras, la familia, el paciente y los proveedores sanitarios sobre qué es lo más apropiado. Es posible evitar muchos de estos conflictos teniendo claro quién es el que toma las decisiones. Una orientación general nos la da el marco legal y ético español. En principio, en lo que afecta a su vida, las decisiones las debe tomar el paciente. Este principio, denominado de autonomía, es una de las piedras angulares de la ética medica en nuestro país ${ }^{(1)}$ Para decidir correctamente, el paciente necesita información sin sesgos. En algunas ocasiones el médico debe participar más o menos en la decisión por problemas diversos, entre los que están; la misma comunicación (sordera por ejemplo), la comprensión (pacientes con limitado conocimiento de palabras que no tienen posibilidad de simplificación), la dificultad para entender todas las consecuencias de aceptar o declinar el tratamiento sugerido, la incapacidad de razonar, los estados depresivos que inclinan a decisiones negativas o de pánico y la desesperación, que bloquean la capacidad de decir de los pacientes con cáncer. En otras ocasiones, el enfermo no quiere asumir la responsabilidad y delega, casi siempre tácitamente, en el profesional sanitario o en grupos familiares que tampoco se atreven a decidir. Finalmente, al tratar de informar a un individuo sobre los riesgos y beneficios estadísticos, el médico o cirujano que asesora al paciente puede verse influido inconscientemente por su visión personal del concepto de calidad de vida aceptable, por el temor a la posible inconsistencia del paciente o sus familiares en asumir el desenlace desfavorable de las decisiones delegadas en los médicos, aun cuando se les hubiese dado toda la información posible, y también por otras circunstancias de su entorno profesional, como la presión del proveedor de servicios por unos objetivos de eficiencia que no tiene en cuenta el mayor consumo de recursos, particularmente de estancias, de los pacientes mayores o funcionalmente límites, o la reacción de otros profesionales sanitarios del equipo ante el impacto emocional por la muerte o discapacidad severa secundaria a un procedimiento, aun como es este caso, siendo la única opción de curación del paciente. Todas estas circunstancias hacen que sea deseable intentar definir objetivamente, como afectará la cirugía del cáncer a la capacidad funcional del sujeto y a la calidad de vida relacionada con la salud (QOL) ${ }^{1}$.

1 Aunque en este texto utilizaremos la abreviatura QOL de "quality of life" en vez de la más precisa HR-QOL de "health related quality of life" por simplicidad. 


\section{¿QUÉ ES LA CALIDAD DE VIDA RELACIONADA CON LA SALUD?}

Para la mayoría de las personas el término "calidad de vida" tiene un significado difícil de definir con precisión relacionado con la satisfacción global con su propia vida. En el ámbito de la salud se aplica específicamente a aqueIlos aspectos de la vida del sujeto que se ven más afectados por del estado de salud o de enfermedad, y se puede definir como "El grado en que el bienestar físico, emocional o social acostumbrado o esperado por el paciente se afecta por un proceso médico o por su tratamiento"(2-5). Por lo tanto, la QOL representa una valoración subjetiva. Distintos sujetos en la misma situación objetiva de salud pueden discrepar en su QOL, debido a diferencias individuales en las expectativas vitales o en la capacidad para afrontar la enfermedad $^{(2-5)}$. La QOL debe evaluarla el paciente y no el médico o terceras personas. Otro componente importante de la QOL es la poli-dimensionalidad, existiendo consenso en que al menos influyen siete aspectos:

1. El bienestar físico y material. entendido como la ausencia de síntomas desagradables; dolor, disnea, náuseas, de miedo a perder la salud y la seguridad económica.

2. El estado funcional en cuanto a la capacidad para llevar a cabo actividades comunes o posibles antes de enfermar.

3. El bienestar emocional, es decir, la combinación de estados afectivos positivos (felicidad, paz espiritual/ aceptación) o negativos (tristeza y ansiedad).

4. El bienestar familiar, es decir la capacidad de mantener las relaciones y la comunicación con la familia.
5. La integración social, o sea la capacidad de mantener y disfrutar de actividades sociales.

6. La satisfacción con el cuidado médico incluyendo la disponibilidad, la posibilidad de afrontar el coste, las molestias y la amabilidad de quienes lo aplican.

7. La sexualidad/intimidad, incluyendo las preocupaciones sobre la imagen física.

\section{¿POR QUÉ ES ÚTIL MEDIR LA CALIDAD DE VIDA EN LA CIRUGÍA DEL CÁNCER DE PULMÓN?}

En medicina, los resultados terapéuticos se han medido tradicionalmente por sus efectos sobre la función, la anatomía y la supervivencia. Sin embargo en el concepto global de salud entran otras dimensiones, que tienen también importancia para los pacientes y deben ser tenidas en cuenta a la hora de tomar decisiones, una de ellas es la QOL.

1. Comparación entre tratamientos. La medición de la QOL en los ensayos clínicos en los que se comparan dos o más tratamientos sirve para determinar el beneficio global, particularmente cuando el tratamiento y los efectos secundaros son considerables. Aunque en oncología la variable primordial sea la supervivencia, los distintos tratamientos pueden suponer:

a) Una ventaja de supervivencia clara con mayor QOL.

b) Similar ventaja de supervivencia pero mayor QOL.

c) Menos supervivencia con más QOL.

d) Más supervivencia y menos QOL.

Está claro que la QOL es la variable decisiva en la opción "b" y en el caso de las opciones " $c$ " $y$ " $d$ " también con- 
tribuye a la decisión, según como el tratamiento modifique la supervivencia y la QOL.

Por tanto, siempre es importante evaluar la QOL de las intervenciones, pero la información que proporciona es particularmente relevante cuando los resultados terapéuticos son pobres y el tratamiento presenta importantes efectos secundarios, como en el cáncer de pulmón en pacientes límite. Incluso en tratamientos eficaces, la evaluación de la calidad de vida QOL puede ser un factor pronóstico, ayudando a decidir el tipo de intervención quirúrgica y como veremos más adelante ayudando también en el manejo. Por eso sorprende la relativa escasez de información sobre la QOL que encontramos en los estudios sobre cirugía del cáncer de pulmón.

2. Mejorar la atención global de paciente: debido a que la valoración de la QOL proporciona una información más detallada de los síntomas, y la perspectiva del paciente sobre el impacto sobre su vida de la enfermad y del tratamiento, su uso en la práctica habitual puede mejorar la evaluación de las necesidades de tratamiento sintomático, rehabilitación o cuidados paliativos. En particular, la información sobre QOL puede revelar ansiedad, depresión o persistencia de síntomas como el dolor o disnea, particularmente cuando son leves o moderados, a los que el paciente se resigna o no quiere manifestar por no defraudar a su médico o por considerarlos tolerables. Por ello, sirve de herramienta facilitadora de la comunicación del paciente con quienes le cuidan sobre posibles intervenciones terapéuticas, psicológicas o sociales para mejorar el bienestar del paciente en estos aspectos. Cuando se consideran tratamientos que prolongan la vida o decisiones sobre el final de la vida, es necesario considerar la valora- ción de cada individuo de lo que hace que la vida merezca la pena, pudiendo ser atractivo un tratamiento que mejore la calidad de vida aunque la reduzca.

3. Factor pronóstico. La QOL puede también ser un factor pronóstico del tratamiento. Como ejemplo los pacientes con cáncer de pulmón, donde la QOL predice la probabilidad de respuesta objetiva con quimioterapia y el cambio de QOL entre el inicio y las 6 semanas de tratamiento es un factor pronóstico de supervivencia ${ }^{(6,7)}$, por lo que sería relevante incluirla como factor de estratificación en las asignaciones aleatorias de los ensayos clínicos.

\section{¿QUÉ INSTRUMENTOS EXISTEN?}

Instrumentos genéricos de salud: son aplicables a todas las poblaciones con o sin enfermedades. Proporcionan puntos de referencia para comparar grupos diversos como poblaciones sanas y enfermas o diferentes grupos de edad. De este tipo son, por ejemplo, el "Nottingham Health profile" (NHP) ${ }^{(8)}$ y el "Short-form-36" (SF36) ${ }^{(9)}$ el "Sickness impact profile" (SIP) ${ }^{(10,11)}$ o el EuroQol (EQ5D) ${ }^{(12)}$ "Nottingham Health profile" (NHP) y el "Short-form-36" (SF36) o su modelo abreviado el SF-12.

Instrumentos genéricos de enfermedad; están diseñados para valorar la QOL de individuos con enfermedades concretas (p.e. EPOC, cáncer, diabetes), tipos específicos de tratamiento (p.e. quimioterapia, transplante), o determinados síntomas (p.e. náusea, incontinencia urinaria). En comparación con otros tipos de instrumentos, estas medidas proporcionan valoraciones más detalladas de las enfermedades, tratamientos o síntomas para los que están diseñados. Es probable que también sean más sensibles a cambios relacionados con el tratamien- 
to. Se destaca el "Functional Assessment of Cancer Therapy: General" (FACT-G) ${ }^{(13)}$ y su versión para cáncer de pulmón el "Functional Assessment of Cancer Therapy - Lung" (FACT-L) ${ }^{(14)}$ o el "European Organization for the Research and Treatment of Cancer Quality of Life Questionnaire" (EORTC QLQ-C30)(15,16) y su suplemento para enfermedades pulmonares "European Organization for the Research and Treatment of Cancer Quality of Life Questionnaire and Lung Cancer Module" (QLQLC13) ${ }^{(17)}$ y el Lung Cancer Symptom Sca$\mathrm{le}^{(18)}$

\section{LÍMITES FUNCIONALES DE LA RESECCIÓN PULMONAR Y RELACIÓN DE LAS VARIABLES FUNCIONALES CON LA CALIDAD DE VIDA POSTOPERATORIA}

Desde principio de los años 80 se demostró que se puede realizar neumonectomías, lobectomías y segmentectomías o resecciones en cuña, con un riesgo aceptable (mortalidad menor del $5 \%$ ) con $\mathrm{FEV}_{1}$ mayor de $2 \mathrm{I}$, mayor de 1,2 I y mayor de $0,6 \mathrm{I}$, respectivamente ${ }^{(19)}$. Dos series recientes, una retrospectiva de 1222 casos y con 186 sujetos cuyo $\mathrm{FEV}_{1}$ era menor de $60 \%{ }^{(20)}$, y otra prospectiva, sugieren que es seguro operar sujetos con $\mathrm{FEV}_{1}>60 \%$ y practicar lobectomías en estadios I y II en sujetos con $\mathrm{FEV}_{1}>50 \%$ si la perfusión de la zona a resecar no supera el $15 \%{ }^{(21)}$, y que en estos pacientes la proporción de sujetos que quedan con insuficiencia respiratoria crónica es baja. Sin embargo, debido el infausto pronóstico del cáncer de pulmón, variaos grupos han operado pacientes con función pulmonar mucho más bajas. En primer lugar hay en los casos en que el tumor se encuentra en zonas de enfisema y su resección se puede considera como una cirugía de reducción de volumen ${ }^{(22-26)}$. Ésta se tole- ra razonablemente bien con funciones preoperatorias muy bajas con una $\mathrm{FEV}_{1}$ $<30 \%$ y la difusión de monóxido de carbono $\left(\mathrm{T}_{\mathrm{L}} \mathrm{CO}\right)<35 \%$, mejorando incluso la función pulmonar(22-26), la capacidad de esfuerzo ${ }^{(26)}$ y la $\mathrm{QOL}^{(26)}$. Es preciso recordar que la reducción de volumen se ha practicado con una mortalidad aceptable (12\%) en pacientes con EPOC y $\mathrm{FEV}_{1}$ y $\mathrm{T}_{\mathrm{L}} \mathrm{CO}>20 \%{ }^{(27)}$ en los que mejora la QOL medida por el SF-36 y el "Chronic Respiratory Diseases Questionnaire" $(C R D Q)^{(28)}$. Sin plantearse la cirugía del cáncer como cirugía de resección de volumen, varios autores han observado que la función pulmonar postoperatoria incluso mejora o no disminuye en la medida prevista en los pacientes con EPOC a los que se les practican lobectomías o resecciones sublobares ${ }^{(21,29-34)}$, ciertas evidencias sugieren que los pacientes que probablemente no sufrirían pérdida funcional son los que tienen un $\mathrm{FEV}_{1} /$ FVC preoperatorio $<55 \%{ }^{(32)}$ o una TLC > 120\% ${ }^{(35)}$.

En algunos estudios se ha intervenido a pacientes con función pulmonar muy baja, así Magdeleinat et al $^{(35)}$ operaron 106 sujetos con un $\mathrm{FEV}_{1}$ preoperatorio del $40 \%$, de los que 11 tenían menos del $30 \%$ y 39 entre $30 \%$ y $39 \%$, y orientaron la resección en base a las predicciones postoperatorias (PPO) de un $\mathrm{FEV}_{1}<30 \%$, una presión arterial pulmonar (PAP) con oclusión de la arteria pulmonar del pulmón a resecar $<25 \mathrm{mmHg}$, y sin desaturación arterial de oxígeno durante dicha oclusión. y en función del estado general, comorbilidades y posible predomino del pulmón afectado. 70 pacientes sufrieron complicaciones y en ellos la estancia se prolongó a 20 días, y 22 necesitaron ventilación mecánica más de 2 días, con una media de 11 días. La supervivencia a largo plazo fue de $82 \%$ a un año y 33\% a los 5 años. Dos pacientes murieron de insuficiencia respi- 
ratoria tardía $(2 \%)$ a los 5 y 31 meses y 11 requirieron oxígeno (11\%), el "estado funcional" evaluado subjetivamente era peor que el preoperatorio en el $20 \%$ de los que sobrevieron 18 meses. En otra cohorte se describen 100 pacientes con $\mathrm{FEV}_{1}<35 \%$, algunos de ellos en tratamiento con oxigenoterapia domiciliaria y 22 con $\mathrm{FEV}_{1}<20 \%$. En 8 se consideró la resección como reducción de volumen y 83 se operaron por vidiotoracoscopia (VATS). La mortalidad fue del $1 \%$ y la morbilidad de $36 \%$. Un $4 \%$ sufrieron insuficiencia respiratoria y un $1 \%$ requirieron ventilación mecánica domiciliaria, y finalmente 11 recibieron una prescripción de oxígeno tras la intervención. No se valoraron a largo plazo, pero es de suponer, por la experiencia en la enfermedad pulmonar obstructiva crónica (EPOC), que estos sujetos tuviesen una pobre calidad de vida. En otro estudio prospectivo de 85 pacientes con $\mathrm{FEV}_{1}$ entre $0,5 \mathrm{~L}$ y $1,2 \mathrm{~L}$ (mediana de $1 \mathrm{~L}$ ) y capacidad de difusión de $\mathrm{CO}$ pulmonar entre 22 y $104 \%$ (mediana $60 \%$ ) y una mediana de $\mathrm{FEV}_{1}$ - $\mathrm{PPO}$ de $34 \%$, la mortalidad global a 5 años fue del $44 \%, 54 \%$, 33\% y $21 \%$ respectivamente para los estadios I, II y III. Además, el 8\% de los pacientes necesitaron oxigenoterapia crónica, todos ellos con un $\mathrm{FEV}_{1}$-PPO $<43 \%$.

Un par de estudios anecdóticos ${ }^{(36,37)}$ sugieren que un consumo de oxígeno preoperatorio máximo por encima de $15 \mathrm{ml} / \mathrm{min} / \mathrm{kg}$ predeciría los pacientes con $\mathrm{FEV}_{1}-\mathrm{PPO}<35 \%$ que no sufrirían insuficiencia respiratoria. Sin embargo, en otro estudio el $\mathrm{FEV}_{1}-\mathrm{PPO}>40 \%$ identificaba a aquellos pacientes que no sufrían insuficiencia respiratoria a largo plazo, mientras que en los que el $\mathrm{FEV}_{1}$ PPO era $<40 \%$ (7 sujetos) el consumo de oxigeno estimado postoperatorio < $10 \mathrm{ml} / \mathrm{min} / \mathrm{kg}$ no permitió identificar a los que requirieron oxigenoterapia ${ }^{(38)}$. Finalmente otros estudios demuestran que la capacidad de esfuerzo, variable funcional que guarda relación con la actividad de la vida diaria y la dimensión de disnea de algunos cuestionaros de enfermedades respiratorias ${ }^{(39)}$, es difícil de predecir a partir de la capacidad de esfuerzo preoperatorio y la cantidad de tejido a resecar ${ }^{(40-43)}$, salvo en aquellos casos en que esta limitada por la ventilación ${ }^{(44)}$.

En conclusión, aunque aquellos sujetos con un $\mathrm{FEV}_{1}$-PPO $>43 \%$ no sufren complicaciones operatorias, dado el pronóstico infausto de esta enfermedad, se considera que se deben extender los límites a cifras inferiores. En este grupo, no se ha demostrado que ninguna variable funcional permita predecir con suficiente exactitud las complicaciones o efectos secundarios crónicos normalmente asociados a pobre calidad de vida, como la necesidad de oxigenoterapia domiciliaria o una capacidad de esfuerzo reducida. En estudios que la han medido directamente, la QOL postoperatoria medida con el EORTCQLQ-30 se relacionaba con el tamaño de la resección (mejor tras lobectomía que tras neumonectomía) ${ }^{(45)}$. En otro parece que la $\mathrm{QOL}$ tras seis meses desde la cirugía guardaba relación con la $\mathrm{T}_{\mathrm{L}} \mathrm{CO}$ pero no con la distancia recorrida en 6 minutos $^{(46)}$.

\section{QOL EN LA CIRUGíA DEL CÁNCER DE PULMÓN}

El dolor por neuralgia intercostal y la disnea postoperatoria debida a la resección de tejido son causas de discapacidad importantes que podrían condicionar la decisión quirúrgica, particularmente a aquellos en los que las expectativas de supervivencia son menores, como son los pacientes en estadios avanzados, de más edad o con peor función pulmonar ${ }^{(47-48)}$. La cuestión po- 
dría formularse: ¿Merece la pena hacer una resección pulmonar por cáncer de pulmón arriesgándose a una calidad de vida pobre en pacientes en los que la supervivencia esperada es baja?

Aunque ningún estudio se ha planteado esta cuestión tal cual, sí hay en la literatura información relevante que permite extraer algunas conclusiones.

En primer lugar hay que considerar que, al menos antes de pasar por ello, muchos pacientes estarían dispuestos a aceptar tratamientos agresivos o tóxicos si les prolonga la supervivencia a pesar de beneficios marginales en la calidad de vida. En un estudio de 388 adultos mayores a los que se les plantearon 17 situaciones terminales o no terminales prefirieron, en general, la prolongación de la supervivencia incluso cuando la calidad de vida que se les proponía era muy pobre por disnea, inmovilidad o dependencia extrema de otros. En la decisión influía la religiosidad, los valores y el miedo a morir ${ }^{(49)}$. Otro factor que parece influir en el deseo de aceptar tratamientos agresivos es el nivel de bienestar. En una serie, los sujetos que consideraban que tenían un alto nivel de bienestar social eran más propensos a aceptar tratamientos agresivos ${ }^{(30)}$.

Por otro lado, Dale et al ${ }^{(51)}$ valoraron la QOL antes de la intervención y a los $1,3,6$ y 9 meses de la misma en una cohorte de 117 sujetos consecutivos sometidos a toracotomía por posible cáncer de pulmón. Mientras que el $14 \%$ de los pacientes referían disnea moderada o intensa antes de la intervención, esta cifra llegó al $34 \%$ al $3^{\text {er }}$ mes, volviendo al $10 \%$ al 6 y 9 meses. De forma similar, las actividades de la vida diaria estaban reducidas en el $11 \%$ de los pacientes en el preoperatorio, proporción que pasó al $21 \%$ a los 3 meses y volviendo a las cifras preoperatorias a los 6 y 9 meses. Se compararon los que se con- firmó que tenían cáncer $(\mathrm{n}=91)$ con los que no $(n=26)$, y se observó que los pacientes con cáncer tenían la misma QOL preoperatoria, pero se deterioraron más tras la intervención.

Zieren et al ${ }^{(52)}$ evaluaron a 52 pacientes a los 12 meses de la intervención haciéndose en 20 pacientes una valoración preoperatoria, y luego cada 3 meses tras la resección presuntamente curativa de cáncer pulmonar. La QOL se evaluó con el EORTC QLQ-C30. Además se hizo un a valoración externa de la QOL por un psiquiatra con el índice de Spitzer. Ambas evaluaciones mostraron correlación significativa, pero pobre $(r=0,41)$. La evaluación de QOL del psiquiatra fue más alta que la del paciente. Tras la cirugía la QOL se vio afectada fundamentalmente por las restricciones en las actividades físicas y en menor medida por los factores emocionales. Los pacientes tenían una puntuación de QOL al alta menor que las preoperatoria, pero a los 6 meses se habían recuperado.

Handy et al ${ }^{(46)}$ utilizaron el "quality of life index" y el SF36 antes de la cirugía y a los 6 meses en 131 pacientes sometidos a resección pulmonar. Comparados con sujetos sanos de la misma edad, los pacientes con cáncer de pulmón tenían los índices de QOL más bajos. A los 6 meses, la QOL era peor que antes de la intervención, fundamentalmente a expensas de los índices de función social y de dolor. Un dato interesante es que la mortalidad quirúrgica intrahospitalaria fue de $5,8 \%$, y un $12,2 \%$ adicional murió en los primeros 6 meses. $\mathrm{Ni}$ el $\mathrm{FEV}_{1}$ ni la prueba de marcha de 6 minutos predijeron la QOL postoperatoria, sólo la $\mathrm{T}_{\mathrm{L}} \mathrm{CO}$ prevista guardaba correlación con la QOL a los 6 meses. En este estudio se observó que a los pacientes les preocupaba más como la cirugía iba a cambiar sus vidas que la mortalidad postoperatoria. 
En otro estudio muy reciente ${ }^{(53)}$ se compararon los resultados de QOL de 132 pacientes operados de carcinoma broncogénico que al menos habían sobrevivido un año (mediana 23 meses) con 121 pacientes operados de by-pass aorto-coronario. Aunque ambos grupos tenían puntuaciones de calidad de vida (SF-36) inferiores a la población sana, no un hubo diferencias entre ellos salvo en el dominio de "función física", pero no en el de ansiedad o depresión. Aunque la puntuación del SF36 bajó a los 3 meses, se habían recuperado los niveles basales a los 12 meses. Los resultados de este estudio son algo sorprendentes, ya que el dolor persistente tras la intervención se debe fundamentalmente a la compresión de los nervios intercostales por los separadores, comprobándose en un estudio previo que un $12 \%$ de los pacientes sometidos a toracotomía tenían dolor torácico frente a un $1 \%$ de los sometidos a esternotomía media ${ }^{(54)}$.

Por otro lado, se ha descrito que los pacientes operados de cáncer de pulmón puntuaban peor su QOL que los de colon y de próstata(55).

Sarna et al ${ }^{(56)}$ midieron la QOL en 142 sujetos con cáncer de pulmón que habían vivido más de 5 años (QOL-survivor y SF36). La mayoría de los supervivientes (71\%) se describieron como esperanzados y el $50 \%$ veían el cáncer como una experiencia positiva, siendo la raza y el "humor alterado" los factores que se correlacionaban con una baja QOL. Este estudio muestra un cuadro sorprendentemente positivo ya que muchos pacientes mostraban una calidad de vida razonablemente elevada incluso a pesar de que la mayoría tenía alteraciones funcionales pulmonares. En los que se observaban alteraciones de la QOL, la depresión, potencialmente mejorable, y no la limitación física, parecía la causa principal. En comparación con otras en- fermedades respiratorias crónicas, los supervivientes de cáncer de pulmón parecen estar en mejor situación mental.

Li et $\mathrm{al}^{(57)}$ estudiaron pacientes intervenidos por cáncer de pulmón en China y que había sobrevivido a largo plazo (mediana de 37 meses en el grupo con VAT y 39 en el de toracotomía). El grupo de VAT era de 27 pacientes y el de toracotomía de 24. La QOL se midió con la versión china del EORTC QLQ-C30 y el EORTC QLQ-LC13. Ambos grupos tenían puntuaciones de QOL similares aunque los intervenidos por VAT tenían mejores puntuaciones en la función pulmonar y menos síntomas, sin que las diferencias alcanzasen significación estadística. La puntuación en la función fue elevada a pesar de una frecuencia bastante elevada de síntomas.

Sugiura et $\mathrm{al}^{(58)}$ estudiaron 44 pacientes con cáncer de pulmón en estadio I.A, a 22 se les intervino por VAT y a 22 por toracotomía. El dolor agudo fue valorado por la necesidad de uso de opiáceos o analgesia epidural y la QOL media preguntando al paciente por dolor torácico crónico, por la limitación del a función del brazo, por el tiempo que tardaron en recuperar la actividad preoperatoria y por la satisfacción con la intervención. Los pacientes intervenidos con VATS tuvieron menos dolor agudo postoperatorio, tardaron menos en recuperar su actividad, les preocupaba menos la cicatriz y tenían mejor impresión global de la intervención.

De la información proporcionada en estos estudios parece deducirse las siguientes conclusiones:

1) Los pacientes con cáncer de pulmón puntúan su QOL más bajo que los no enfermos de la misma edad, pero no que los pacientes que van a ser sometidos a otras cirugías importantes. 
2) La QOL se deteriora tras la intervención, particularmente durante los 3 a 6 primeros meses, en relación con el dolor y la perdida de capacidad funcional.

3) Tras 3-6 meses los pacientes recuperan la QOL. Es posible que esto ocurra porque la neuralgia secundaria a la contusión de los nervios intercostales mejore en ese plazo, porque la función pulmonar y capacidad de esfuerzo se recupere durante ese periodo $^{(43)}$, e incluso porque los pacientes en peor situación funcional no superen dicho periodo ${ }^{(46)}$. En los que sobreviven a 1 año o más, la QOL está determinada más por factores psicológicos que funcionales o de dolor, a pesar de que muchos tienen la función pulmonar deteriorada.

4) Parece que a los pacientes les preocupa más como la cirugía va a cambiar su vida que la mortalidad postoperatoria. La mayoría de los pacientes parecen dispuestos a asumir el riesgo si supone vivir más, pero tras la intervención echan de menos que les hubiesen informado sobre los cambios en la capacidad física y en su función social, y de la posibilidad de que estas alteraciones persistan algún tiempo.

5) No parece razonable desaconsejar la cirugía a ningún paciente operable, correctamente informado, que decida asumir la intervención, por la presunción por parte del equipo que le atiende de una mala calidad de vida. Un $10 \%$ de los pacientes con función pulmonar muy baja $\mathrm{FEV}_{1} \mathrm{PPO}<40 \%$ pueden quedar con insuficiencia respiratoria crónica.

6) Existe un periodo entre 30 días y 6 meses que no se consideran en los estudios de riesgo operatorio y en los que podría haber un exceso de mortalidad relacionada con la cirugía y no con el tumor.

7) La intervención por VAT parece mejorar la calidad de vida en los primeros meses pero no a largo plazo Esto se debe en parte a una reducción del dolor. Se debe, por tanto, intentar minimizar la lesión de los nervios intercostales porque es un factor importante asociado al deterioro en la calidad de vida durante los primeros meses de la intervención, y en algunos pacientes, de forma duradera.

8) La medición de la QOL puede facilitar la evaluación por parte de los que cuidan al paciente de sus síntomas (dolor), y de la existencia de problemas corregibles (depresión).

\section{QOL COMO FACTOR PRONÓSTICO}

Aunque la QOL es un factor pronostico importante en el cáncer de pulmón avanzado, en el único estudio en el que se ha aplicado a la resección quirúrgica se mostró una asociación débil entre la QOL y la estancia media, que a su vez se relacionaba con la presentación o no de complicaciones ${ }^{(59)}$. En otro estudio, la valoración geriátrica del paciente mediante cuestionarios se relacionaba con el riesgo de la cirugía, en concreto la baja actividad de la vida diaria, determinada mediante un cuestionario, y la pobre función cognitiva, que se asociaban a una mayor mortalidad ${ }^{(60)}$.

\section{REFERENCIAS BIBLIOGRÁFICAS}

1. LEY 41/2002, de 14 de noviembre, Básica Reguladora de la Autonomía del Paciente y de Derechos y Obligaciones en Materia de Información y Documentación Clínica. Boletín Oficial del Estado de 15.11.02.

2. Bergner M. Quality of life, health status, and clinical research. Med Care 1989; 27: S148-S156. 
3. Cella DF. Quality of life: Concepts and definition. J Pain Symptom Manage 1994; 9:186-92.

4. Mast ME. Definition and measurement of quality of life in oncology nursing research: Review and theoretical implications. Oncol Nurs Forum 1995; 22:95764.

5. Gill TM, Feinstein AR. A critical appraisal of the quality of quality of life measurements. JAMA 1994; 272:619-26.

6. Bonomi P, Kim K, Fairclough D, Cella D, Kugler J, Rowinsky E, Jiroutek M, Johnson D. Comparison of survival and quality of life in advanced non-small-cell lung cancer patients treated with two dose levels of paclitaxel combined with cisplatin versus epitoside with cisplatin: results of an Eastern Cooperative Oncology Group trial. J Clin Oncol 2000; 18:623-31.

7. Eton D, Fairclough DL, Cella D, Yount $\mathrm{SE}$, Bonomi P, Jonhson SH. Early change in patient-reorted health during lung cancer chemotherapy predicts clinical outcomes beyond those predicted by baseline report;: results from eastern cooperative oncology group study 5592. J Clin Oncol 2003;21:1536-43.

8. Hunt SM, McEwen J, McKenna SP. Measuring health stats: a new tool for clinicians and epidemiologists. J Royal Coll Gen Pract 1985;35:185-88.

9. Ware JE Jr, Sherbourne CD. The MOS 36item short-form health survey (SF-36). I. Conceptual framework and item selection. Med Care 1992; 30:473-83.

10. Gilson BS, Gilson JS, Bergner M, Bobbit RA, Kressel S, Pollard WE, Vesselago $M$. The sickness impact profile. Development of an outcome measure of health care. Am J Public Health 1975; 65:130410.

11. Bergner M, Bobbitt RA, Carter WB, Gilson BS. The Sickness Impact Profile: development and final revision of a health status measure. Med Care 1981 19:787805.
12. EuroQol Group. EuroQol-A new facility for the measurement of health-related quality of life. Health Policy 1990; 16: 199-208.

13. Cella DF, Tulsky DS, Gray G, Sarafian B, Linn E, Bonomi A, Silberman M, Yellen SB, Winicour P, Brannon J, et al.. The Functional Assessment of Cancer Therapy Scale: development and validation of the general measure. J Clin Oncol 1993; 11:570-9.

14. Cella DF, Bonomi AE, Lloyd SR, Tulsky DS, Kaplan E, Bonomi P. Reliability and validity of the Functional Assessment of Cancer Therapy -- Lung (FACT-L) quality of life instrument. Lung Cancer 1995; 12:199-220.

15. Aaronson NK, Ahmedzai S, Bergman B, Bullinger $M$, Cull A, Duez NJ, Filiberti A, Flechtner $\mathrm{H}$, Fleishman SB, de Haes JC, et al.. The European Organization for Research and Treatment of Cancer QLQC30: a quality-of-life instrument for use in international clinical trials in oncology. J Natl Cancer Inst 1993; 85:365-76.

16. Osoba D, Aaronson N, Zee B, Sprangers $M$, te Velde A. Modification of the EORTC QLQ-C30 (version 2.0) based on content validity and reliability testing in large samples of patients with cancer. The Study Group on Quality of Life of the EORTC and the Symptom Control and Quality of Life Committees of the $\mathrm{NCl}$ of Canada Clinical Trials Group. Qual Life Res 1997; 6:103-8.

17. Bergman B, Aaronson NK, Ahmedzai S, Kaasa S, Sullivan M. The EORTC QLQLC13: a modular supplement to the EORTCCore Quality of Life Questionnaire (QLQ-C30) for use in lung cancer clinical trials. Eur J Cancer 1994; 30A:635-42

18. Hollen PJ, Gralla RJ, Kris MG, Cox C, Belani CP, Grunberg SM, Crawford J, Neidhart JA. Measurement of quality of life in patients with lung cancer in multicenter trials of new therapies. Psychometric assessment of the Lung Cancer Symptom Scale. Cancer 1994; 73:2087-98. 
19. Miller JI, Grossman GD, Hatcher CR. Pulmonary function test criteria for operability and pulmonary resection. Surg Gynecol Obstet 1981;153:893-5.

20. Licker MJ, Widikker I, Robert J, Frey JG, Spiliopoulos A, Ellenberger C,Schweizer A, Tschopp JM. Operative mortality and respiratory complications after lung resection for cancer: impact of chronic obstructive pulmonary disease and time trends. Ann Thorac Surg. 2006;81:1830-7.

21. Santambrogio L, Nosotti M, Baisi A, Ronzoni G, Bellaviti N, Rosso L. Pulmonary lobectomy for lung cancer: a prospective study to compare patients with forced expiratory volume in $1 \mathrm{~s}$ more or less than $80 \%$ of predicted. Eur J Cardiothorac Surg. 2001; 20(4):684-7.

22. McKenna RJ Jr, Fischel RJ, Brenner $M$, Gelb AF. Combined operations for lung volume reduction surgery and lung cancer. Chest. 1996; 110:885-8.

23. DeRose JJ Jr, Argenziano M, El-Amir N, Jellen PA, Gorenstein LA, Steinglass KM, Thomashow B, Ginsburg ME. Lung reduction operation and resection of pulmonary nodules in patients with severe emphysema. Ann Thorac Surg. 1998; 65:314-8.

24. Edwards JG, Duthie DJ, Waller DA. Lobar volume reduction surgery: a method of increasing the lung cancer resection rate in patients with emphysema. Thorax. 2001; 56:791-5.

25. Choong CK, Meyers BF, Battafarano RJ, Guthrie TJ, Davis GE, Patterson GA,Cooper JD. Lung cancer resection combined with lung volume reduction in patients with severe emphysema. J Thorac Cardiovasc Surg. 2004; 127:132331.

26. Pompeo E, De Dominicis E, Ambrogi V, Mineo D, Elia S, Mineo TC. Quality of life after tailored combined surgery for stage I non-small-cell lung cancer and severe emphysema.Ann Thorac Surg. 2003; 76:1821-7.
27. Fishman A, Martinez F, Naunheim K, Piantadosi S, Wise R, Ries A, Weinmann G,Wood DE; National Emphysema Treatment Trial Research Group. National Emphysema Treatment Trial Research Group. A randomized trial comparing lung-volume-reduction surgery with medical therapy for severe emphysema. $\mathrm{N}$ Engl J Med, 2003; 348:2059-73.

28. Miller JD, Berger RL, Malthaner RA, Celli $\mathrm{BR}$, Goldsmith $\mathrm{CH}$, Ingenito $\mathrm{EP}$, Higgins D, Bagley P, Cox G, Wright CD. Lung volume reduction surgery vs medical treatment: for patients with advanced emphysema. Chest, 2005; 127:1166-77.

29. Carretta A, Zannini P, Puglisi A, Chiesa G, Vanzulli A, Bianchi A, Fumagalli A, Bianco $S$. Improvement of pulmonary function after lobectomy for non-small cell lung cancer in emphysematous patients. Eur J Cardiothorac Surg. 1999; 15:602-7.

30. Sekine Y, Iwata T, Chiyo M, Yasufuku K, Motohashi S, Yoshida S et al Minimal alteration of pulmonary function after lobectomy in lung cancerpatients with chronic obstructive pulmonary disease. Ann Thorac Surg. 2003; 76:356-61

31. Korst RJ, Ginsberg RJ, Ailawadi M, Bains MS, Downey RJ Jr, Rusch VW et al Lobectomy improves ventilatory function in selected patients with severe COPD. Ann Thorac Surg. 1998; 66:898-902.

32. Baldi S, Ruffini E, Harari S, Roviaro GC, Nosotti M, Bellaviti N et al. Does lobectomy for lung cancer in patients with chronic obstructive pulmonary disease affect lung function? A multicenter national study. J Thorac Cardiovasc Surg. 2005; 130:1616-22

33. Martin-Ucar AE, Nakas A, Pilling JE, West $\mathrm{KJ}$, Waller DA. A case-matched study of anatomical segmentectomy versus lobectomy for stage I lung cancer in high-risk patients. Eur J Cardiothorac Surg. 2005; 27:675-9.

34. Keenan RJ, Landreneau RJ, Maley $\mathrm{RH}$ Jr, Singh D, Macherey R, Bartley S et al. 
Segmental resection spares pulmonary function in patients with stage I lung cancer. Ann Thorac Surg. 2004; 78:228-33

35. Magdeleinat $P$, Seguin A, Alifano $M$, Boubia S, Regnard JF. Early and long-term results of lung resection for non-smallcell lung cancer in patients with severe ventilatory impairment. Eur J Cardiothorac Surg. 2005; 27:1099-105

36. Morice RC, Peters EJ, Ryan MB, Putnam JB, Ali MK, Roth JA. Exercise testing in the evaluation of patients at high risk for complications from lung resection. Chest. 1992;101:356-61

37. Bolliger CT, Soler M, Stulz P, Gradel E, Muller-Brand J, Elsasser $S$ et al. Evaluation of high-risk lung esection candidates: pulmonary haemodynamics versus exercise testing. A series of five patients. Respiration. 1994; 61:181-6.

38. Beccaria M, Corsico A, Fulgoni P, Zoia MC, Casali L, Orlandoni G et al. Lung cancer resection: the prediction of postsurgical outcomes should include long-term functional results. Chest 2001; 120:37-42.

39. Ruiz de Ona Lacasta JM, Puente Maestu L, Rodriguez Hermosa JL, Tatay Marti E, Cubillo Marcos JM. [A comparison of several measurement scales for assessing dyspnea in their daily activities in patients with chronic obstructive lung disease] Arch Bronconeumol. 2000; 36:25-8.

40. Bolliger CT, Wyser C, Roser H, Soler M, Perruchoud AP. Lung scanning and exercise testing for the prediction of ostoperative performance in lung resection candidates at increased risk for complications. Chest. 1995; 108:341-8.

41. Bobbio A, Chetta A, Carbognani P, Internullo E, Verduri A, Sansebastiano G, et al. Changes in pulmonary function test and cardio-pulmonary exercise capacity in COPD patients after lobar pulmonary resection. Eur J Cardiothorac Surg. 2005; 28:754-8.
42. Puente Maestu L, Rodriguez Hermosa JL, Ruiz de Ona JM, Santa-Cruz Seminiani A, de Lucas Ramos P, Garcia de Pedro J, et al. [Role of postoperative estimate of maximum oxygen uptake in predicting cardiorespiratory insufficiency in the immediate postoperative period in thoracic surgery] Arch Bronconeumol. 1998; 34:127-32.

43. Bolliger CT, Jordan $\mathrm{P}$, Soler M, Stulz $\mathrm{P}$, Tamm M, Wyser C et al. Pulmonary function and exercise capacity after lung resection. Eur Respir J. 1996; 9:415-21.

44. Puente-Maestu L, Ruiz de Ona JM, Rodriguez-Hermosa JL, Santa-Cruz Siminiani A, Tatay E, Cubillo JM. [Prediction of exercise capacity after lung resection in patients with chronic airflow limitation] Arch Bronconeumol. 1998; 34:473-8.

45. Hendriks J, Van Schil P, Van Meerbeeck J, Gdeedo A, Van Marck E, Vanmaele $\mathrm{R}$, et al. Short-term survival after major pulmonary resections for bronchogenic carcinoma. Acta Chir Belg. 1996; 96:2739.

46. Handy JR Jr, Asaph JW, Skokan L, Reed CE, Koh S, Brooks G et al. What happens to patients undergoing lung cancer surgery? Outcomes and quality of life before and after surgery. Chest. 2002; 122:2130.

47. Varela G, Novoa N, Jimenez MF. Influence of age and predicted forced expiratory volume in $1 \mathrm{~s}$ on prognosis following complete resection for non-small cell lung carcinoma. Eur J Cardiothorac Surg. 2000; 18:2-6.

48. Myrdal G, Lambe M, Gustafsson G, Nilsson K, Stahle E Survival in primary lung cancer potentially cured by operation: influence of tumor stage and clinical characteristics.Ann Thorac Surg. 2003; 75:356-63.

49. Yellen SB, Cella DF. Someone to live for: social well-being, parenthood status, and decision making in oncology. J Clin Oncol 1995; 13:1255-62. 
50. Cicirelli VG. Relationship of psychosocial and background variables to older adults' end-of- life decisions. Phsychol Aging 1997; 12:72-9.

51. Dales RE, Belanger R, Shamji FM, Leech J, Crepeau A, Sachs HJ. Quality-of-life following thoracotomy for lung cancer. J Clin Epidemiol. 1994; 47:1443-9.

52. Zieren HU, Muller JM, Hamberger U, Pichlmaier H. Quality of life after surgical therapy of bronchogenic carcinoma. Eur J Cardiothorac Surg. 1996; 10:233-7.

53. Myrdal G, Valtysdottir S, Lambe M, Stahle E. Quality of life following lung cancer surgery. Thorax. 2003; 58:194-7.

54. Asaph JW, Handy JR Jr, Grunkemeier GL, Douville EC, Tsen AC, Rogers RC et al. Median sternotomy versus thoracotomy to resect primary lung cancer: analysis of 815 cases. Ann Thorac Surg. 2000; 70:3739.

55. Schag CAC, Ganz PA, Wing DS, Sim MS, Lee JJ. Quality of life in adult survivors of lung, colon, and prostate cancer. Qual Life Res 1994; 3:127-41.
56. Sarna L, Padilla G, Holmes C, Tashkin D, Brecht ML, Evangelista L. Quality of life of long-term survivors of non-small-cell lung cancer. J Clin Oncol. 2002; 20:2920-9.

57. Lee TW, Lam SS, Ng CS, Sihoe AD, Wan IY, Yim AP. Quality of life following lung cancer resection: video-assisted thoracic surgery vs thoracotomy. Chest. 2002; 122:584-9.

58. Sugiura $H$, Morikawa T, Kaji M, Sasamura Y, Kondo S, Katoh H. Long-term benefits for the quality of life after video-assisted thoracoscopiclobectomy in patients with lung cancer. Surg Laparosc Endosc Percutan Tech. 1999; 9:403-8.

59. Parsons JA, Johnston MR, Slutsky AS. Predicting length of stay out of hospital following lung resection using preoperative health status measures Qual Life Res. 2003;12(6):645-54.

60. Fukuse T, Satoda N, Hijiya K, Fujinaga T. Importance of a comprehensive geriatric assessment in prediction of complications following thoracic surgery in elderly patients. Chest. 2005; 127:886-91. 
